\title{
Opposing temperature dependence of the stretching response of single PEG and PNiPAM polymers
}

\author{
Adrianna Kolberg, ${ }^{\dagger}$ Christiane Wenzel, ${ }^{\dagger}$ Klara Hackenstrass, ${ }^{\dagger}$ Richard Schwarzl, ${ }^{\dagger}$ Christian \\ Rüttiger, ${ }^{\S}$ Thorsten Hugel, ${ }^{*, \dagger, \perp}$ Markus Gallei, ${ }^{\S, \|}$ Roland R. Netz ${ }^{*, *}$ and Bizan N. Balzer*, ${ }^{* \dagger}$ \\ 'Institute of Physical Chemistry, Albert-Ludwigs-Universität Freiburg, Albertstr. 23a, 79104 Freiburg, Germany \\ Department Institute of Theoretical Bio- and Soft Matter Physics, Freie Universität Berlin, Arnimallee 14, 14195 \\ Berlin, Germany \\ ${ }^{\S}$ Ernst-Berl-Institute of Technical and Macromolecular Chemistry, Technische Universität Darmstadt, Alarich- \\ Weiss-Str. 4, 64287 Darmstadt, Germany \\ ${ }^{\perp}$ Cluster of Excellence livMatS @ FIT - Freiburg Center for Interactive Materials and Bioinspired Technologies, \\ University of Freiburg, Georges-Köhler-Allee 105, 79110 Freiburg, Germany \\ "Organic Macromolecular Chemistry, Saarland University, Campus Saarbrücken C4 2, 66123 Saarbrücken, Germany
}

KEYWORDS. Stimuli responsive polymer, single molecule force spectroscopy, atomic force microscope, molecular dynamics simulations.

\begin{abstract}
The temperature response of switchable polymer blends and coatings is important for the development of high performance materials. Although this is well studied for bulk materials, a proper understanding on the molecular level, in particular for high stretching forces, is still missing. Here we investigate the molecular details of the temperature response of two widely used watersoluble polymers, namely poly ( $N$-isopropylacrylamide) (PNiPAM) and polyethylene glycol (PEG) with a combined approach using atomic force microscopy (AFM) based single molecule force spectroscopy (SMFS) experiments and molecular dynamics (MD) simulations. SMFS became possible by the covalent attachment of long and defined single polymers featuring a functional end group. Most interestingly, varying the temperature has contrasting effects for PEG and PNiPAM. This on first sight surprising result can be understood with the help of MD simulations in explicit water. We find that hydration is widely underestimated for the mechanics of macromolecules and that a polymer chain has competing energetic and entropic elastic
\end{abstract}


components. We propose to use the temperature dependence to quantify the energetic behaviour for high stretching forces. This fundamental understanding of temperature dependent single polymer behavior might lead to new innovations like fast switchable polymer blends and coatings with antagonistically acting polymer chains.

\section{Introduction}

Polymer coatings, blends and composites are common in every household and became a part of our every-day life in the last century. They are fundamental for industrial applications due to their unique molding ability, their robustness and their light weight. ${ }^{1-3}$ Even though we face all their advantages on a daily basis, their behavior is still not completely understood. In particular, their response to external stimuli like force or temperature changes is often only understood phenomenologically.

The temperature response of polymers is important for both, technical applications and fundamental understanding of polymer physics. ${ }^{4-8}$ Depending on the technical application, polymer properties have to be maintained over a large temperature range (as e.g. in cars), or coil to globule transitions are used to obtain stimuli responsive materials (e.g. for triggered drug release). ${ }^{9-11}$ In polymer physics, temperature is a fundamental parameter. Therefore, the dependence of polymer properties on temperature is crucial for a thorough understanding of polymer mechanics.

Atomic force microscopy (AFM) based single molecule force spectroscopy (SMFS) is a versatile tool to investigate temperature dependent single polymer mechanics in liquid environment, in particular when combined with molecular dynamics (MD) simulations. ${ }^{12-19}$ Here, we focus on two widely used water-soluble polymers with different temperature response, namely poly $(N$ isopropylacrylamide) (PNiPAM) and polyethylene glycol (PEG).

In bulk experiments PNiPAM undergoes in water a transition from a coil to globular conformation at its lower critical solutions temperature (LCST) of around $305 \mathrm{~K} \cdot{ }^{20,21}$ This LCST is close to the temperature at which most physiological processes occur, which makes PNiPAM promising for the development of controlled drug delivery systems. ${ }^{22-25}$ The coil conformation of PNiPAM below the LCST is thought to be stabilized via formation of hydrogen bond bridges between water molecules and the amide side groups. ${ }^{26}$ The water molecules align around the hydrophobic 
backbone making the polymer soluble in water and leading to energetic stabilization. At temperatures above the LCST, the entropy of the polymer-water system dominates, making the exothermic formation of hydrogen bonds unfavorable. Thus, the bound water molecules are released to increase their entropy and the polymers collapse into a globular state. ${ }^{27}$ It still remains unclear, if this is a bulk effect, or if this can already be observed for a single polymer chain. The single molecule stretching behavior at different temperatures has been measured by AFM, but with controversial results. Kutnyanszky et al. have found a linear temperature response without any sign of a sharp transition or minimum around the $\operatorname{LCST} .{ }^{28}$ Cui et al. claimed that the stretching force has a minimum at the LCST. ${ }^{16}$ Liang et al. found a transition of the force-extension profile from worm-like chain behavior to a Rayleigh-Plateau of constant force at the LCST ${ }^{17}$ Furthermore, Zhang et al. have investigated the solvent dependent single molecule stretching behavior of PNiPAM, observing thermally induced multisite adsorption above its LCST. ${ }^{18}$ The first study was restricted to a small temperature range of $299-313 \mathrm{~K}$, while the other three studies use the nanofishing method for their AFM-based experiments, where a polymer physisorbed on a surface, e.g. glass or $\mathrm{Au}(111)$, is randomly picked up with a cantilever tip. This leads to several problems that interactions between different polymer chains physisorbed on the substrate cannot be excluded (bulk effects) and that every time a different polymer might be picked. These are problematic points, as discussed later, and caused us to revisit PNiPAM's temperature behavior.

PEG is a linear macromolecule, consisting of $-\left(\mathrm{CH}_{2}-\mathrm{CH}_{2}-\mathrm{O}\right)$ - repeating units. It is used for medical and technological purposes. ${ }^{29-32}$ Unlike most polymers, PEG is generally soluble in water even for a high degree of polymerization. ${ }^{33}$ PEG does not exhibit a LCST transition and on first sight looks like an ideal entropic elastic spring. This has indeed been confirmed in hexadecane, where the force-extension relation of PEG is in accordance with the freely-jointed chain model (FJC). ${ }^{34,35}$ In water the situation changes, PEG does no longer show the characteristics of an ideal entropic spring at high stretching forces. ${ }^{34,19}$ The reason is found in the structural change from gauche to trans. ${ }^{35}$ The ratio of monomers in the trans and gauche conformation during stretching and the number of water bridges was extracted from water-explicit MD-simulations. Altogether, a dominant solvent related effect of energy over entropy was suggested at high stretching forces. ${ }^{19}$ Again experimental temperature dependent behaviour shall clarify these points.

Here, we compare the force response of PEG and PNiPAM at various temperatures in a combined experimental and theoretical study. This allows us to delineate the molecular details of the different 
temperature responses of PEG and PNiPAM. While PEG becomes softer at higher temperatures, PNiPAM stiffens with increasing temperature. These at first sight contradictory results can be explained in molecular detail with our combined approach. We anticipate that this will help guiding the development of tailored materials that have to function over a wide force and temperature range.

\section{Methods}

Chemicals The chemicals used for cleaning of glass wear were Ammonia solution (Roth, Karlsruhe, Germany, 28.0 - $30.0 \%$ ) and hydrogen peroxide solution (Sigma Aldrich, St. Louis, MO, USA, $\geq 30 \%$ ) as RCA solution with ultrapure water (Purelab Chorus 1, Elga LabWater, Celle, Germany, 18.2 $\mathrm{M} \Omega \mathrm{cm}$ ) with a ratio of 1:1:5. For the functionalisation process toluene (Fisher Chemicals, Hampton, NH, USA, $99.99 \%$ ), ethanol (Roth, Karlsruhe, Germany, z99.9\%), HEPES buffer (Pan-Reac AppliChem, Darmstadt, Germany, 99.5 \%, 10 mM, pH = 7, $50 \mathrm{mM} \mathrm{NaCl}$ ) silanePEG-mal (NANOCS, Boston, MA, USA, $\left.M_{\mathrm{w}}=5 \mathrm{kDa}, l_{\text {cont }}=41 \mathrm{~nm}\right)$ and HEPES-buffer $(10 \mathrm{mM}$ HEPES, NaCl $50 \mathrm{mM}$; pH 7) were used. For the synthesis of PNiPAM all solvents and reagents were purchased from Alfa Aesar (Haverhill, MA, USA), Sigma-Aldrich (St. Louis, MA, USA), Fisher Scientific (Hampton, NH, USA), ABCR (Karlsruhe, Germany) and used as received unless otherwise stated. Deuterated solvents were purchased from Sigma-Aldrich. $N$-isopropylacrylamid (NiPAM) was recrystallized out of toluene and $n$-hexane $(1: 1)$ and dried in vacuum. $\mathrm{Cu}^{(\mathrm{I})} \mathrm{Br}$ was washed five times with glacial acetic acid and ethanol. Tris[2-(dimethylamino)ethyl]amine (Me ${ }_{6}$ TREN), $N, N$-dimethyl formamide (DMF), 2 bromo-iso-butyric tert-butylester (tBbiB) were stored under argon atmosphere.

Polymers The polymer used for the PEG experiments was purchased as Thiol-PEG-Thiol (HSPEG-SH, Creative PEGWorks, NA, USA, $M_{\mathrm{w}}=35 \mathrm{kDa}$ ). The expected mean contour lengths for the experiments was calculated including the silane-PEG-mal linker with a monomer length of $0.356 \mathrm{~nm}$ and a molecular weight of $44.05 \mathrm{Da}$ to $282 \mathrm{~nm} .{ }^{35,19}$ Adding the silane-PEG-mal linker length with about $41 \mathrm{~nm}$ leads to a total length of $323 \mathrm{~nm}$.

PNiPAM was synthesized as follows. The corresponding reaction path can be found in Figure S1a: $9.00 \mathrm{~g}(79.53 \mathrm{mmol}) \mathrm{NiPAM}$ and $3.20 \mu \mathrm{L}(0.018 \mathrm{mmol}) t \mathrm{BbiB}$ were placed in a Schlenk tube and $18 \mathrm{~mL}$ of a 50:50 mixture of ultrapure water and DMF were added. The mixture was degassed twice and the polymerization was started by adding $90 \mu \mathrm{L}(0.018 \mathrm{mmol})$ of a $0.2 \mathrm{M}$ solution of 
$\mathrm{Cu}^{\mathrm{I}}\left(\mathrm{Me}_{6} \mathrm{TREN}\right) \mathrm{Br}$ in DMF. After $40 \mathrm{~min} 12 \mathrm{mg}(0.075 \mathrm{mmol})$ potassium ethyl xanthogenate were added. After $10 \mathrm{~min}$ the reaction was cooled in an ice bath for $30 \mathrm{~min}$. The mixture was diluted with $160 \mathrm{~mL}$ THF and filtered through an aluminium column to remove the residual copper catalyst. The polymer was precipitated in $800 \mathrm{~mL}$ diethyl ether and dried in vacuum (yield: $4.06 \mathrm{~g}$, $\left.M_{\mathrm{n}}: 497 \mathrm{kDa}, \emptyset=1.28\right)$.

$500 \mathrm{mg}(0.001 \mathrm{mmol})$ of the CTA-endcapped PNiPAM were dissolved in $20 \mathrm{~mL}$ ultrapure water and $100 \mathrm{mg}(2.64 \mathrm{mmol}) \mathrm{NaBH}_{4}$ were added. After $2 \mathrm{~h}$ the polymer/solvent mixture was dialyzed against water for 4 days. The polymer was obtained by lyophilization (452 mg). $300 \mathrm{mg}$ of the polymer were dissolved in $10 \mathrm{~mL}$ DMF and $45 \mathrm{mg}(0.16 \mathrm{mmol})$ tris(2-carboxyethyl)phosphine hydrochloride (TCEP) were added. After $24 \mathrm{~h}$ the mixture was diluted with water and dialyzed against water for 4 days again. $243 \mathrm{mg}$ of the resulting polymer were obtained after lyophilisation $\left(M_{\mathrm{n}}: 510 \mathrm{kDa}, \emptyset=1.28\right.$, see Figure $\left.\mathrm{S} 1 \mathrm{~b}\right)$.

For PNiPAM, the monomer length was calculated with respect to the backbone using the $\mathrm{C}-\mathrm{C}$ bond length of $154 \mathrm{pm}$ and a bond angle of $109.5^{\circ} .{ }^{36}$ With a calculated monomer length of around $252 \mathrm{pm}$ and a monomer weight of $113 \mathrm{Da}$, the average contour length could be calculated to $1.14 \mu \mathrm{m}$ with 4513 repeating units. Adding the silane-PEG-mal linker length with about $41 \mathrm{~nm}$ leads to a total length of $1.18 \mu \mathrm{m}$.

Polymer characterisation Standard size-exclusion chromatography (SEC) was performed with a system composed of a 1260 IsoPump G1310B (Agilent Technologies, Santa Clara, CA, USA), a 1260 VW detector G1314F at $254 \mathrm{~nm}$ (Agilent Technologies) and a 1260 RI-detector G1362A at $35{ }^{\circ} \mathrm{C}$ (Agilent Technologies), DMF (with $\mathrm{LiCl}, 1 \mathrm{~g} / \mathrm{L}$ ) as the mobile phase (flow rate $1 \mathrm{~mL} / \mathrm{min}$ ) on a GRAM column set for DMF (at $50{ }^{\circ} \mathrm{C}$ ) from PSS (Polymer Standard Service (PSS), Mainz, Germany) (GRAM 30, GRAM 1000, GRAM 1000). Calibration was carried out using PMMA standards for DMF (from PSS). Samples were measured with concentrations between $1 \mathrm{mg} / \mathrm{mL}$ and $3 \mathrm{mg} / \mathrm{mL}$. For data acquisition and evaluation of the measurements, PSS WinGPC® UniChrom 8.2 was used.

NMR spectra were recorded on a Bruker DRX 500 spectrometer (Billerica, MA, USA) working at $500 \mathrm{MHz}$. NMR chemical shifts were referenced relative to the used solvent $\left(\mathrm{D}_{2} \mathrm{O}\right)$. For data acquisition and evaluation of the measurements, NMR software MestReNova ${ }^{\circledR} 11.0$ was used. 
Simulation details All molecular dynamics simulations are performed with the GROMACS simulation package (version 4.6 .5 or newer). ${ }^{37}$ The time step is set to $2 \mathrm{fs}$. The temperature is set to a fixed value for each individual simulation. For temperature and pressure coupling, the v-rescale and Parinello-Rahman algorithms are used. ${ }^{38,39}$ The pressure is isotropic and set to 1 bar with a water compressibility of $4.5 \times 10^{-5} \mathrm{bar}^{-1}$. All simulations are performed with periodic boundary conditions in all three directions. The cut-off of nonbonded interactions is set to $1.0 \mathrm{~nm}$. The particle mesh Ewald method is used for the long-range electrostatic interactions. ${ }^{40}$

For PEG the force field charmm35r is used. ${ }^{41}$ The elongated $\left(\mathrm{H}-\left[\mathrm{CH}_{2}-\mathrm{O}-\mathrm{CH}_{2}\right]_{12}-\mathrm{H}\right)$ chain is placed in a $3 \mathrm{~nm} \times 3 \mathrm{~nm} \times 9.6 \mathrm{~nm}$ box with 2900 tip3p water molecules. To equilibrate the system, the initial energy minimization of the system is followed by a 10 ps NVT simulation with constant volume and without pressure coupling and a 2 ns NPT simulation with an isotropic pressure of 1 bar. For the production run, the first and the last oxygen atoms are defined as pulling groups. A constant force between $1 \mathrm{pN}$ and $600 \mathrm{pN}$ is applied in $\mathrm{z}$ direction. For low forces, we additionally performed simulations using a longer chain $\left(\mathrm{H}-\left[\mathrm{CH}_{2}-\mathrm{O}-\mathrm{CH}_{2}\right]_{24}-\mathrm{H}\right)$ placed in a $4.5 \mathrm{~nm} \times 4.5 \mathrm{~nm} \times 20 \mathrm{~nm}$ box with 13433 water molecules. Each pulling simulation is performed for at least $200 \mathrm{~ns}$. The temperature remains unchanged throughout a single simulation run and is set to $250 \mathrm{~K}, 300 \mathrm{~K}, 325 \mathrm{~K}, 350 \mathrm{~K}$ and $400 \mathrm{~K}$, respectively.

For simulations of PNiPAM, parameters remain the same as for PEG if not stated otherwise. For PNiPAM we use a recently modified version of the OPLS-AA force field with partial charges optimized by comparison to quantum mechanical simulations together with the SPC/E water model. ${ }^{42-45}$ The first and the last $\mathrm{C}$ atom of the backbone of $\mathrm{H}-\left[\mathrm{C}_{6} \mathrm{H}_{11} \mathrm{NO}\right]_{20}-\mathrm{H}$, which are adjacent to the side-chain of the polymer, are defined as pulling groups. A constant force between $1 \mathrm{pN}$ and $500 \mathrm{pN}$ is applied in $z$ direction. We simulate isotactic (meso-diad) PNiPAM, where the side chains are located on one side of the backbone, and syndiotactic (racemo-diad) PNiPAM, where the side chains are on alternating sides of the chain. The elongated chain is placed in a $3 \mathrm{~nm} \times 3 \mathrm{~nm} \times 11 \mathrm{~nm}$ box with $3121 \mathrm{SPC} / \mathrm{E}$ water molecules. Each pulling simulation is performed for at least $1000 \mathrm{~ns}$. The temperature remains unchanged throughout a single simulation run and is set to $288 \mathrm{~K}, 298 \mathrm{~K}$, $308 \mathrm{~K}$ and $318 \mathrm{~K}$, respectively.

For both PNiPAM and PEG, the extension in the pull direction is calculated as the time average of the separation in $z$ direction of the two pulling groups over the course of a simulation. The extension 
of PEG is normalized by the contour length $L_{\mathrm{C}, 0}=11 \times 0.356 \mathrm{~nm}$ or $L_{\mathrm{C}, 0}=23 \times 0.356 \mathrm{~nm}$, depending on the polymer length and in accordance to Liese et al. ${ }^{19}$ For isotactic PNiPAM the contour length used for normalization is $L_{\mathrm{C}, 0}=19 \times 0.266 \mathrm{~nm}$ and for syndiotactic PNiPAM $L_{\mathrm{C}, 0}=19 \times 0.264 \mathrm{~nm}$ in accordance to Kanduč et $a l .^{44}$

AFM tip functionalisation The covalent attachment of one single polymer to a cantilever tip is decisive for single molecule force experiments. It enables a high reproducibility when performing force spectroscopy with a certain polymer on a specific cantilever tip. Influences on the measurements like differences in the contour length due to different attachment points to the cantilever tip, variation of the spring constant of the cantilever or interactions with further polymers can be widely prevented. ${ }^{46}$ Furthermore, a high yield of single molecule events can be obtained (42\% for PNiPAM and $19 \%$ for PEG).

Silicon nitride AFM cantilevers, namely MLCT and MLCT-BIO-DC (both: Bruker AFM probes, Camarillo, CA, USA) were used for all measurements. First, the cantilevers were activated with oxygen plasma to gain hydroxyl groups on the surface of the cantilever tip. While the MLCT probes were treated for 1 min with $20 \%$ power, the thermally more stable MLCT-BIO-DC probes were treated for 2 min with $40 \%$ power, both with a pressure of $0.1 \mathrm{mbar}$. As a next step, a $5 \mathrm{kDa}$ silanePEG-mal (NANOCS, Boston, MN, USA) linker was bound to the cantilever tip. The linker enabled to couple a probe molecule to the tip via a covalent bonding. Therefore, the cantilevers were incubated in a solution of silane-PEG-mal in toluene $\left(1.25 \mathrm{mg} / \mathrm{ml}, 3 \mathrm{~h}, 60{ }^{\circ} \mathrm{C}\right) .{ }^{47}$ Even though the cantilever tip is covered with maleimide groups, these undergo a hydrolysis in water (inactive PEGs) leaving just few binding sites for the single probe polymer to be attached. ${ }^{48}$ The inactive PEGs serve as a passivation layer to reduce undesirable interaction between the cantilever tip and the surface as well as between the single PEG polymer and the cantilever tip. For functionalisation with PNiPAM, the PEGylated cantilevers were rinsed in toluene and ethanol before incubation in a solution of PNiPAM-SH in ethanol $(1.25 \mu \mathrm{g} / \mathrm{ml}, 3 \mathrm{~h}, \mathrm{RT})$. For PEG, the PEGylated cantilevers were rinsed in toluene and incubated in a SH-PEG-SH toluene solution $\left(1.25 \mu \mathrm{g} / \mathrm{ml}, 1 \mathrm{~h}, 60^{\circ} \mathrm{C}\right)$. After a final rinsing, the cantilevers were stored in HEPES buffer at $4{ }^{\circ} \mathrm{C}$ until use in the AFM experiment. 
For every functionalisation, control cantilevers were additionally prepared by the same procedure except just pure solvent was used instead of a SH-PEG-SH or PNiPAM-SH solution. A scheme of an AFM based single molecule force spectroscopy setup can be found in Figure 1 .

AFM Measurements All measurements were performed with a Cypher ES (Asylum Research, an Oxford Instruments company, Santa Barbara, CA, USA) using a heating and cooling sample stage for temperature variation. All measurements took place in ultrapure water on a silicon oxide wafer cleaned in ethanol using a sonicator (Elmasonic S15, Elma, Singen, Germany). Before every measurement, the inverse optical lever sensitivity (InvOLS) was determined by fitting a linear function to the repulsive regime of a force-extension curve. In order to reduce errors, the determination of InvOLS was performed by using an average of at least five individual InvOLS values. The spring constant of the cantilever was determined by the thermal noise method. ${ }^{49}$

The measurement parameters were defined as followed: force distance: $1-3 \mu \mathrm{m}$, velocity: $1 \mu \mathrm{m} / \mathrm{s}$, trigger point: $500 \mathrm{pN}$, sampling rate: $5 \mathrm{kHz}$ and dwell time toward the surface: $0-1 \mathrm{~s}$. To minimize the influence of the probe, force-extension curves were recorded in a grid like manner with $10 \times 10$ points, covering $20 \times 20 \mu \mathrm{m}^{2}$ (force maps). At least two force maps were obtained per cantilever, with and without dwell. The temperature was varied in a random order. Following a temperature change, force-extension curves were collected after a stabilisation time of at least $5 \mathrm{~min}$. Prior to a series of measurements, at least one control cantilever was measured on different spots to ensure the cleanliness of the $\mathrm{SiO}_{\mathrm{x}}$-surface and a contamination free functionalisation. If the control cantilevers showed an absence of stretch events, the PNiPAM or PEG functionalized cantilevers were measured, respectively.

For data evaluation, a self-programmed evaluation software based on IgorPro (Wavemetrics, Portland, OR, USA) was used. The force-extension curves were corrected for drift by fitting a linear function to the baseline after the last stretch. Then, the linear function was subtracted from the force-extension curve leading to curves such as shown in Figures 1a and $b$.

Even though we used PNiPAM polymers with a low polydispersity index value $\bigoplus=1.28$, significant differences in the polymer lengths were observed in the AFM experiments. This observation can be explained by different phenomena, which have already been described in literature before. ${ }^{19}$ The common method to detect the molecular weight and the polydispersity is size-exclusion chromatography (SEC), which determines a relative weight of the polymer compared to standards 
like polystyrene or poly(methyl methacrylate).$^{50}$ As polymers can significantly differ with respect to their hydrodynamic volume compared to these standards, a deviation of the determined molecular weight of PNIPAM from the absolute molecular weight is expected. Another important influence is given by the silicon layer. As the functionalisation process is performed in air, spurious water might start an oligomerisation of the silane molecules prior to the attachment to the tip. This leads to a flexible silane network with fewer anchor points. ${ }^{51} \mathrm{~A}$ further explanation is related to the position of the anchoring point of the polymer to the cantilever tip, since the polymer is not necessarily bound to the apex. ${ }^{19}$ As the cantilever detects only a force component in vertical direction, a non-vertical orientation of the polymer between a pin point on the substrate and the cantilever tip might lead to a shift of the detected force according to Serr et al. ${ }^{52}$ Yet, for long polymers such as the PEG and PNiPAM polymers presented here, the deviation from a vertical orientation is expected to be less than $1^{\circ}$, leading to a force variation below the detection limit of the AFM. ${ }^{53}$ For PEG, a broad mass distribution is expected (PDI is not indicated by the manufacturer). Furthermore, for HS-PEG-SH it is chemically possible that the ends of two polymers oxidise and react with each other to a disulfide bridge. ${ }^{54}$ That might lead to length values which are multiples of the contour length. Even though these phenomena have an influence on the observed absolute contour length of the polymer, they do not affect the stretching response of the respective polymer itself. Therefore, all these effects are not expected to show any dependence on temperature and therefore should not affect the general results found here.

\section{Results and Discussion}

Figure 1 shows a schematic of the AFM-based single molecule force experiments including example traces and simulation snapshots. A single stretch event at a contour length of about $350 \mathrm{~nm}$ such as given in Figure 1a was observed in the PEG data in 95 of the 500 measured force-extension curves (rate of 19\%). For PNiPAM, 252 out of 600 force-extension curves (42\%) were found, such as shown in Figure 1b. In order to discuss the temperature dependence of the force-extension traces, a single master curve for every temperature was determined using only those curves that show a stretch event to at least $500 \mathrm{pN}$ to be in the linear regime of bond stretching (see Figures S2 and S5). Then, the extensions were rescaled to a length $L_{0}$ at a force of $500 \mathrm{pN}$. Finally, the forceextension curves were averaged by a binomial smoothing, where a Gaussian filter convolves the 
data with normalized coefficients derived from Pascal's triangle at a level equal to the smoothing parameter $20 .^{55}$ These rescaled traces are shown in Figure 2.
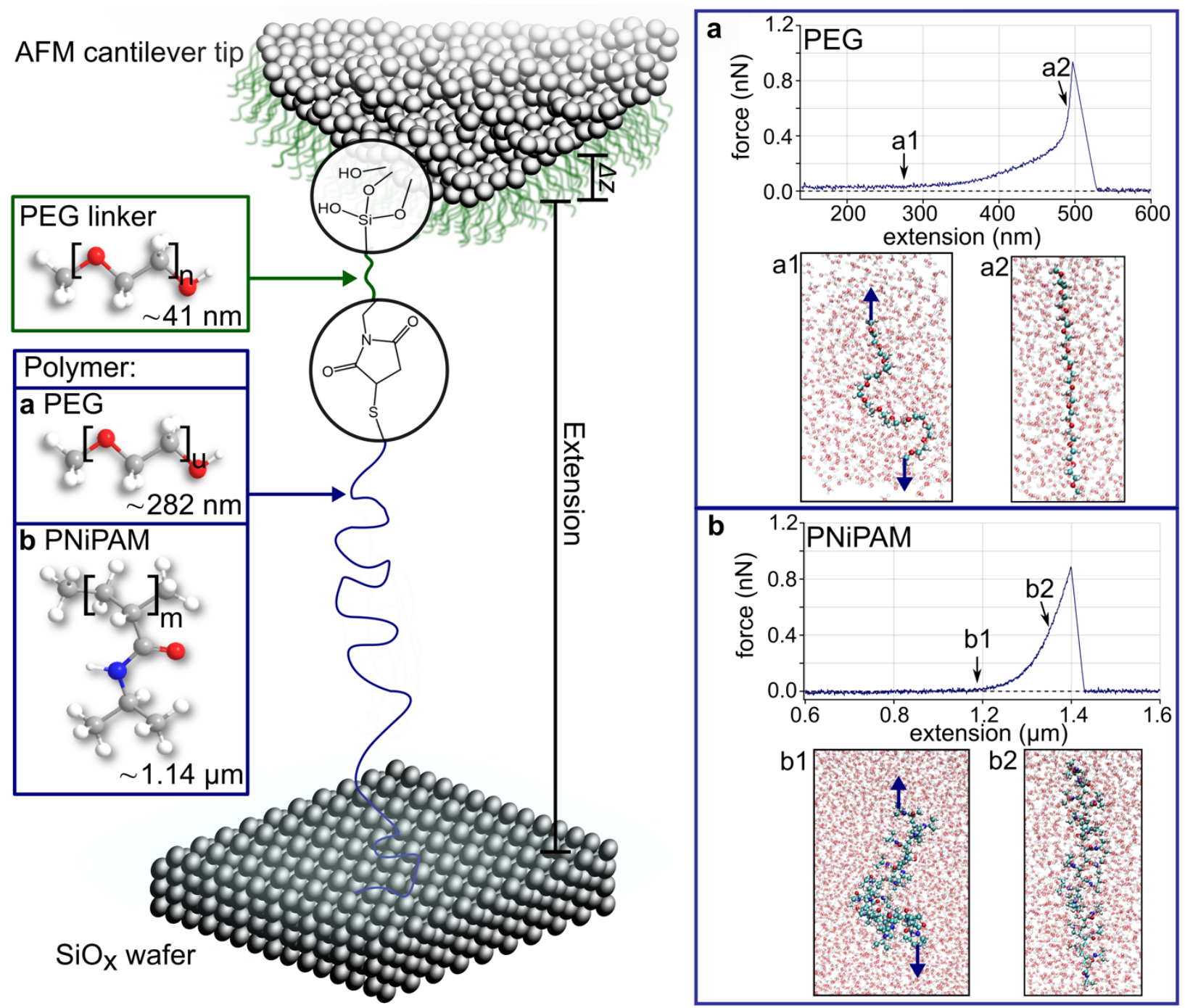

Figure 1. Schematic AFM single molecule setup with a zoom-in to the functional groups (circular frames) and an exemplary monomeric unit for the PEG linker (green box) and the polymer (blue box) at room temperature. On the right part, example force-extension curves for (a) PEG and (b) PNiPAM including snapshots of the MD-simulations at the forces indicated in the force-extension traces are shown at $298 \mathrm{~K}$. These forces are $15 \mathrm{pN}$ and $600 \mathrm{pN}$ for PEG (a1, a2) and $20 \mathrm{pN}$ and $500 \mathrm{pN}$ for isotactic PNiPAM (b1, b2). The monomer numbers are $n=114(41 \mathrm{~nm})$ for the PEG linker, $u=795(282 \mathrm{~nm})$ for the PEG and $m=4513(1.14 \mu \mathrm{m})$ for the PNiPAM polymer. $\Delta z$ indicates a possible vertical shift corresponding to the anchoring position of the polymer on the AFM cantilever tip. 
For the master curves depicted in Figure 2a we used 20 traces (4\%) in total that reached forces of at least $500 \mathrm{pN}$ in order to investigate the polymer behaviour at intermediate and high stretching forces. We focus on the range from 0.4 to $1.0 L_{0}$ because the temperature effect is best seen here. Furthermore, we can be sure that this extension range only comprises the stretching of a single molecule (for raw data see Figure S2). The difference between the force-extension curves for PEG measured at different temperatures (Figure 2a and Figure S3) can be described as a decrease of the force with increasing temperature at a given relative extension, which is discussed in detail below. In other terms, an increase of ca. $5 \%$ of relative extension (at an applied force of $100 \mathrm{pN}$ ) is observed in a temperature range of $278 \mathrm{~K}$ to $318 \mathrm{~K}$. Note that it is crucial to compare the temperature dependence for data taken with one and the same cantilever and for one set of polymers. Otherwise, variations of the spring constant of different cantilevers and possibly the contour length of polymers could mask the temperature effects (see methods section for details). A second data set confirms the observed temperature behavior (Figure S4).

For PNiPAM, the length varies significantly even for a single cantilever tip. In order to minimize the effects of contour length, we only selected traces with contour lengths between $1.0 \mu \mathrm{m}$ and $2.5 \mu \mathrm{m}$ and detachment forces of more than $500 \mathrm{pN}$. This resulted in 20 traces $(3 \%)$, which are shown in the master curves depicted in Figure 2b. The raw data can be found in Figure S5. Additionally, Figure S6 shows all master curves for different temperatures. Again, a temperature dependent shift in the stretching force can be observed, but this time in the opposite direction compared to PEG. The stretching forces increase with increasing temperature, i.e. the chain becomes stiffer, for a given relative extension (see Figure S6 and S7). In other terms, an increase of ca. $1 \%$ of relative extension (at an applied force of $100 \mathrm{pN}$ ) is observed in a temperature range of $278 \mathrm{~K}$ to $328 \mathrm{~K}$. Note, that there is no sudden change in the shape or frequency of the recorded force-extension curves when comparing temperatures below and above the LCST. This agrees with the expectation that a highly stretched single molecule does not show cooperative effects, which are rather expected for weakly stretched chains that can self-interact. This is in contrast to the study by Cui et al., which claimed that the stretching force has a minimum at the LCST when examining the temperature range of $304 \mathrm{~K}$ to $313 \mathrm{~K}$ with a difference of relative extension of up to $10 \%$ at 200 pN. ${ }^{16}$ Furthermore, we did not observe any plateaus in our force-extension curves over the whole range of temperature values from $278 \mathrm{~K}$ to $328 \mathrm{~K}$ such as observed by Liang et al. ${ }^{17}$ 
Yet, both Cui et al. and Liang et al. performed nanofishing experiments. Zhang et al. have already pointed out that AFM cantilever tip functionalization might be a better way to obtain clean and real single molecule stretching events. ${ }^{18}$ Nanofishing might lead to cooperative effects that might strongly affect the outcome of a single molecule study due to additional substrate adsorbed molecules. ${ }^{56}$ Our presented covalent attachment of a single molecule to the cantilever tip, enables to exclude any interactions with neighboring polymers. Therefore, our results are consistent with the study by Kutnyanszky et $a l .^{28}$, where also single molecule attachment to an AFM cantilever tip had been done. Yet, our significantly larger allows us to deduce the molecular mechanism for this behavior. A second data set for PNiPAM taken with a different cantilever confirms the observed temperature dependence (Figure S7). To further understand these observations, MD-simulations were performed and compared to the experimental data.
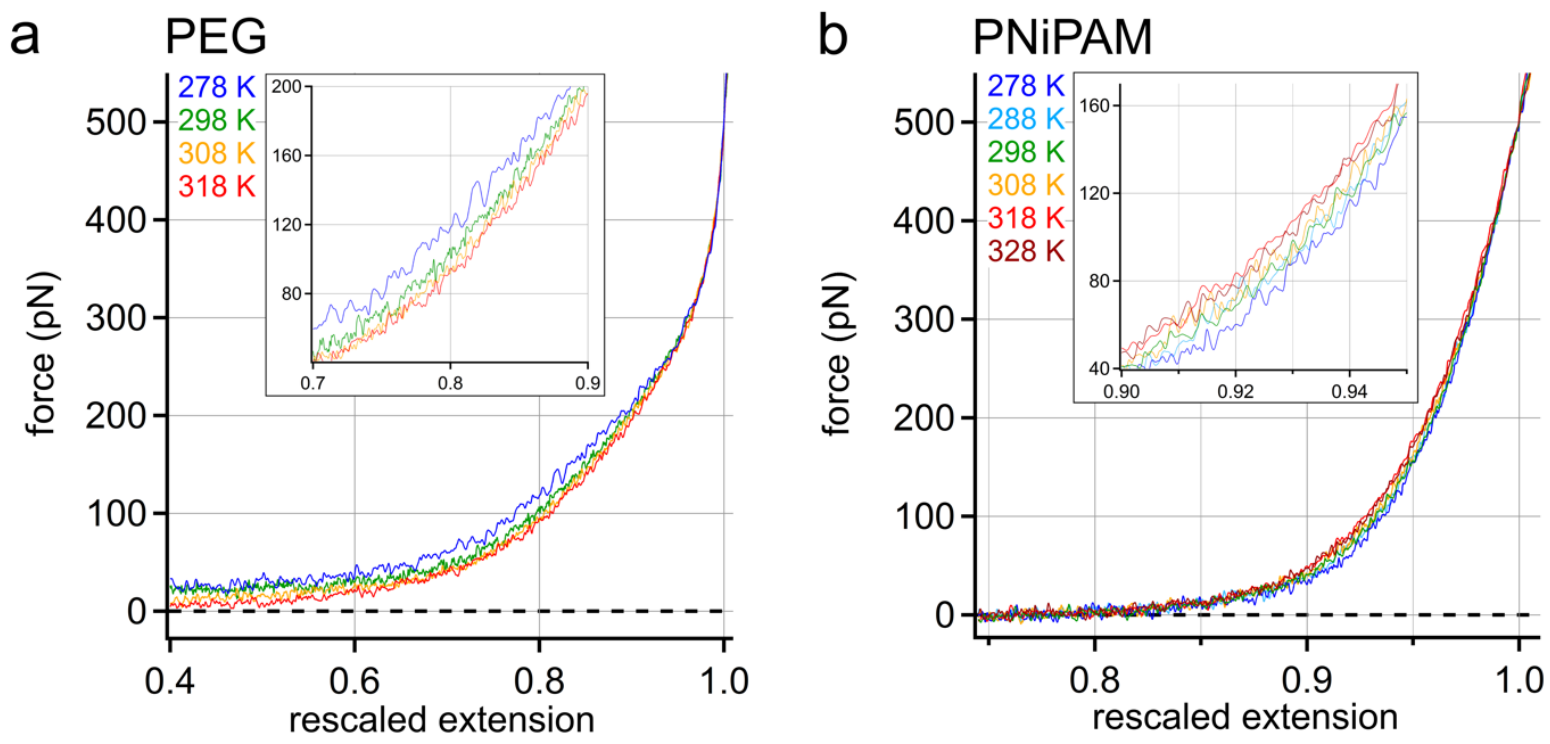

Figure 2. Experimental AFM data: (a) Master curves of PEG showing decreasing stretching force with increasing temperature. (b) Master curves of PNiPAM showing an opposite temperature dependence compared to PEG. A single master curve for every temperature was determined based on force-extension curves with a stretch event to at least $500 \mathrm{pN}$. After rescaling the extensions to the length $L_{0}$ at a force of $500 \mathrm{pN}$, the force-extension curves were averaged by a binomial smoothing.

Figure 3a shows a comparison of the experimental master curves for PEG to the MD-simulation. The distinct tripartite structure with a middle section showing an almost constant force-extension 
slope is well reproduced by the simulation. Also, the temperature shift agrees well with the experimental data. This validation enables us to extract the molecular mechanisms for PEG from the MD simulations (see below).

Figure 3b,c show the comparison of the experimental master curves for PNiPAM with two sets of MD-simulations. As the polymer was synthesized by a controlled radical polymerisation without any manipulation of the propagating chain ends, a predominantly atactic arrangement of the side groups is expected. This is confirmed by the ${ }^{13} \mathrm{C}-\mathrm{NMR}$ data, which shows the expected ratios between the signals in the range of $30-45$ ppm (Figure S1c). ${ }^{57}$ Accordingly, the experimental force-extension curves are between force-extension curves from MD-simulations representing the extreme cases of a fully isotactic and a fully syndiotactic polymer, as shown in Figure $3 \mathrm{~b}$ (isotactic) and Figure 3c (syndiotactic). For the isotactic case, an extremely slow relaxation of the PNiPAM chain leads to a strong variation of the stretching force masking any clear trend with temperature. By contrast, the syndiotactic case comprises small error values and presents a clear force-extension course for the different temperatures. In particular, the temperature shift in the force response shows the same qualitative behavior for both AFM experiment and for the MD simulation, although differing quantitatively. The quantitative difference might result from the difference between atactic (experiment) and syndiotactic (MD simulation) arrangement. 


\section{a PEG}
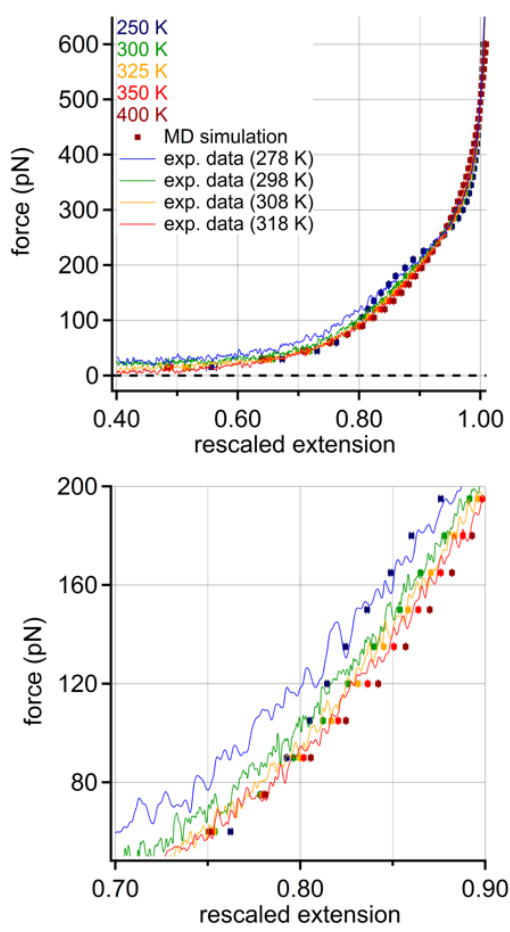

b PNiPAM (isotactic)
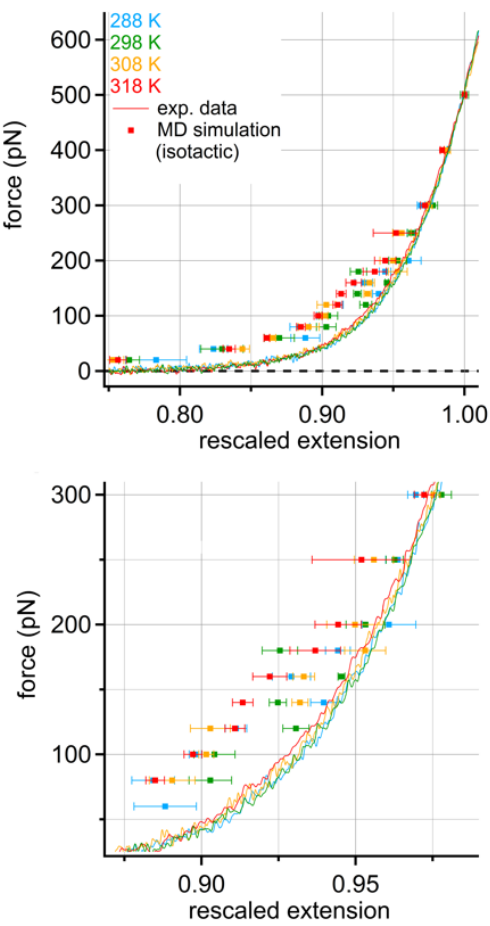

C PNiPAM (syndiotactic)
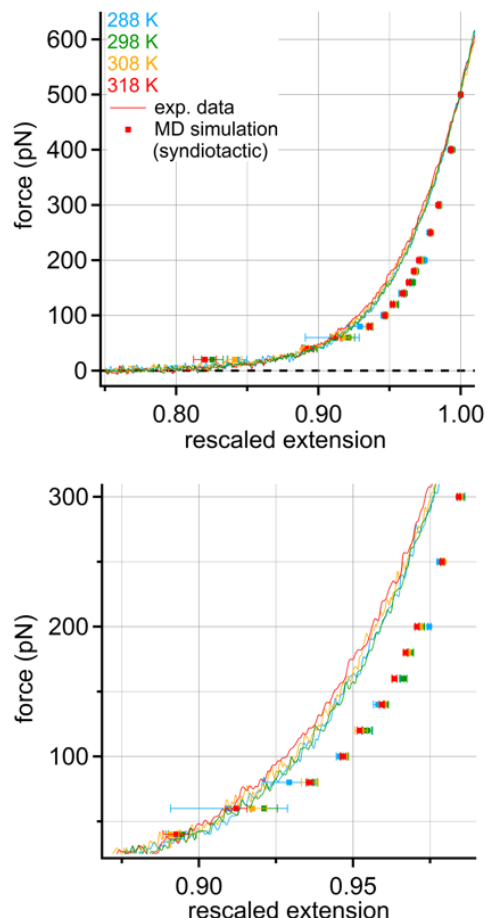

Figure 3. Comparison of MD-simulations with the experimental data: (a) MD-simulations for a PEG molecule for a wide temperature range showing the same characteristics as the experimental data. Note the significantly larger temperature range in the simulations. (b) MD-simulations of an isotactic and (c) of a syndiotactic PNiPAM polymer compared to the experimental data for atactic PNiPAM, respectively. The experimental force-extension trace lies between the MD-simulations of the syndiotactic and isotactic PNiPAM. Also, the magnitude of the temperature induced force shift is in between the two extreme cases considered in the MD-simulations. Respective zoom-ins are shown in the bottom row for better comparison of the relevant mid-force extension range around $200 \mathrm{pN}$.

The temperature dependence of the free energy per length, $F / L_{0}$, can be generally expressed as $T^{n}$ with an exponent $n$. An ideal entropic behavior can be described by the FJC model ${ }^{58}$ :

$$
\frac{z_{\text {ete }}}{L_{c}}=\operatorname{coth} \frac{f b}{k_{B} T}-\frac{k_{B} T}{f b}
$$

while the wormlike chain (WLC) model is not purely entropic: ${ }^{59}$ 


$$
f=\frac{k_{B} T}{l_{p}}\left[\frac{z_{\text {ete }}}{L_{c}}+\frac{1}{4\left(1-\frac{z_{\text {ete }}}{L_{C}}\right)^{2}}-\frac{1}{4}\right]
$$

Here, $z_{\text {ete }}$ is the rescaled end-to-end distance, $L_{\mathrm{c}}$ the rescaled contour length, $b$ the temperature independent Kuhn length (see Table S1), $f$ the applied force and persistence length $l_{\mathrm{p}}=\kappa / k_{B} T$ with the bending rigidity $\kappa$ assumed to be temperature independent. Next, the free energy $F$ is given by:

$$
F=U-T S
$$

While the FJC model leads to $U / F=0$ and $-T S / F=1$, the WLC model leads to $U / F=-1$ and $-T S / F=2$ with the energy $U$ and the entropy $S$ assumed to be independent from $T .{ }^{19}$ While in both cases $T S / F$ is constant, $F / L_{0}$ is proportional to $T^{n}$ with $n=1$ for the FJC model (with temperature independent Kuhn length, see Table S1) and $n=2$ for the WLC model (with temperature independent bending rigidity $\kappa$ ). In contrast, a purely energetic behavior should be temperature independent, i.e. $n=0$. We name regimes with exponents $n>1$ super entropic, $n<0$ super energetic and with $0<n<1$ mixed.

The commonly used FJC and WLC models fail to describe the force-extension behavior of both PEG and PNiPAM over the whole force range (Figure S8 and S9) ${ }^{19}$ In particular, both polymers deviate from the purely entropic spring models at intermediate and highly stretched states. In fact, they represent energetic or mixed springs with different temperature behavior, as discussed in the following.

The temperature dependent force response of the PEG polymer is best analyzed by normalizing the force response with respect to the temperature dependence, i.e. to find the exponent of the temperature $n$ at which the experimental force response for the different temperatures fall on top of each other. In particular, the optimal exponent $n$ is found by the lowest coefficient of variation (ratio of standard deviation and mean value) for the different temperatures at each stretching force value (Figure S10). Thus, the different regimes (entropic and energetic) can be distinguished along the course of the stretching force. In Figure 4 a the stretching free energy, $F$ per $L_{0}$ is shown, which is determined by integration of the master curves (force vs rescaled extension) given in Figures 2 
and 3. Considering the different temperature ranges for the experiment and the simulation, the data agree well. This is more obvious when $F / L_{0}$ is normalized by $k_{\mathrm{B}} T$ (Figure $4 \mathrm{~b}$ ). In particular, for low forces, the MD simulations data is better described by a scaling exponent $n=1$, indicating purely entropic behavior. Above $150 \mathrm{pN}$, a similar trend as for the AFM data is observed where energy dominated behavior is found. Both $F / L_{0}$ and the slope of $F / L_{0}$ vs force is highest for the lowest temperature. $F / L_{0}$ for the various temperatures increases linearly with the force up to $300 \mathrm{pN}$. For force values above $300 \mathrm{pN}$ a sub-linear $F / L_{0}$ vs force relation is observed. While $F / L_{0}$ vs force curves for the lowest temperature $(250 \mathrm{~K})$ show a non-linear course, those for higher temperatures $(300 \mathrm{~K}, 325 \mathrm{~K}, 350 \mathrm{~K}$ and $400 \mathrm{~K})$ show a linear behavior up to $300 \mathrm{pN}$.
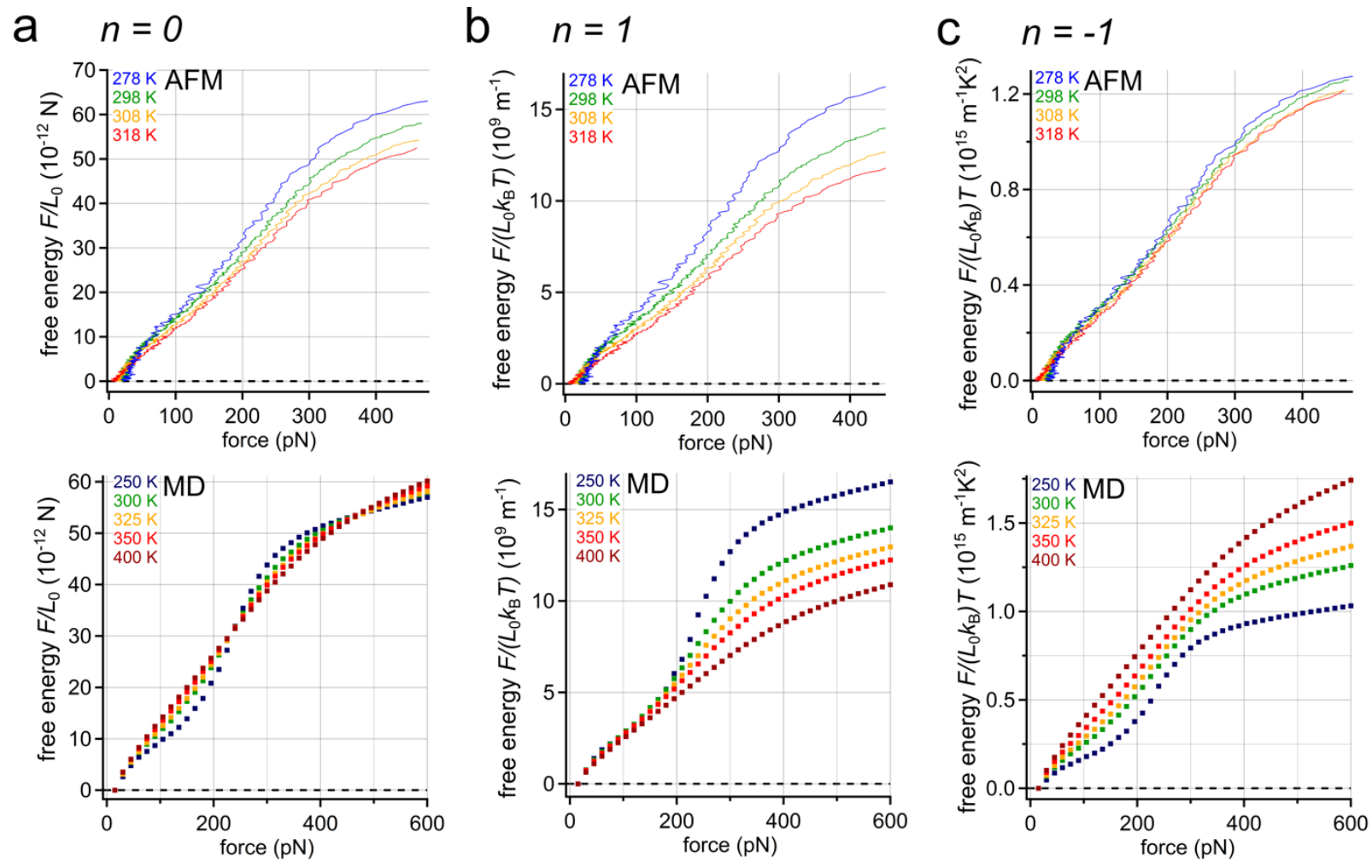

Figure 4. Temperature dependence of the stretching free energy $F$ for experimental AFM data and the MD-simulations of PEG: (a) stretching free energy $F$ per $L_{0}$ (extension at a force of $500 \mathrm{pN}$ ) vs. force (case $n=0)$, (b) $F$ per $L_{0}$ divided by $k_{\mathrm{B}} T(n=1)$, (c) $F$ per $L_{0}$ divided by $k_{\mathrm{B}} T^{-1}(n=-1)$. Note the significantly larger temperature range in the simulations.

For AFM data, an energy dominated behaviour is observed over the whole course of the curve (except below $50 \mathrm{pN}$ ). The AFM curves superimpose even better for a further normalization $F /\left(L_{0} k_{\mathrm{B}}\right) T$ vs force $(n=-1)$ presenting a super energetic regime (Figure $\left.4 \mathrm{c}\right)$. Altogether, the MD 
simulations are best described by an entropy dominated chain at low forces $(n=1)$ and an energetic behaviour $(n=0)$ starting at intermediate forces, while the experiment already shows dominant energetic contributions $(n=-1)$ at all investigated forces.

In order to understand these observations, we examine the mean fraction of gauche and trans states in the PEG chain according to MD simulations. Figure 5a shows the mean fraction of monomers in the trans state for different temperatures as a function of force. The initially shallower increase of $\Phi_{\text {trans }}$ for low temperatures can be explained by a higher number of water bridges forming two hydrogen bonds with the PEG backbone, which we depict in Figure 5b. The decrease in the number of water bridges explains the overall decrease in $F / L_{0}$ with increasing temperature (Figure $4 \mathrm{a}$ ). In addition, the pronounced non-linear behavior of $\Phi_{\text {trans }}$ for low temperatures around $250 \mathrm{pN}$ can explain the increase in the slope of $F / L_{0}$ observed in Figure $4 \mathrm{a}$ at this force. Here, the water bridges stabilize the gauche state, which has a smaller contour length compared to the trans state (see also the increase of temperature dependent contour length using the FJC model, Figure S8). This also explains the temperature dependent cross-over of PEG around $250 \mathrm{pN}$, which can be observed in Figure $4 \mathrm{a}$ and 5. Note that a temperature dependent contour length can be obtained using the FJC model to fit PEG force-extension curves (see Figure S8), if a temperature independent Kuhn length is assumed (see Table S1). Such a temperature dependent contour length would just be a fit parameter with no real physical meaning and we therefore believe that the above discussed exponent $n$ describes the underlying physical processes better. 

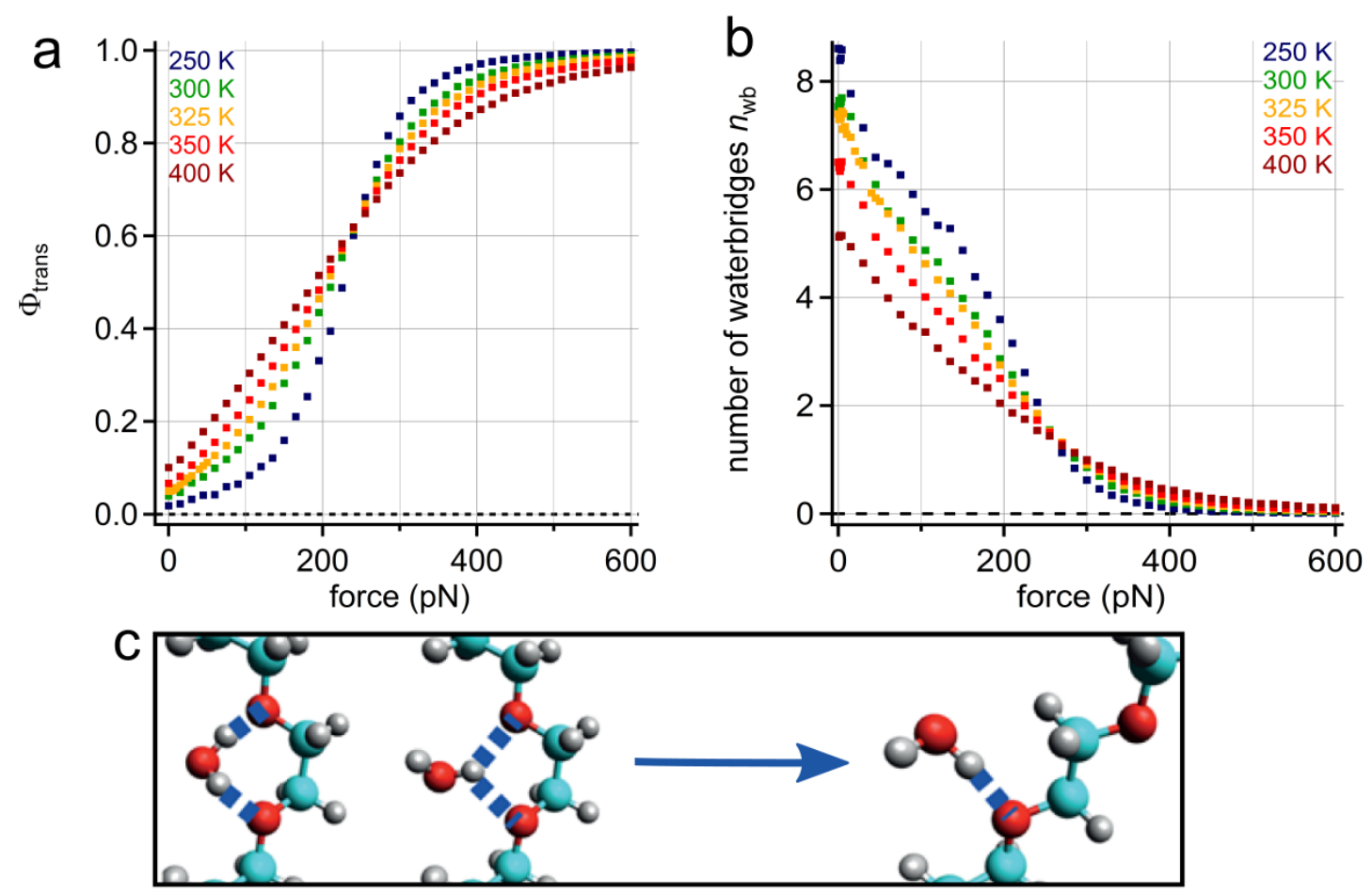

Figure 5. MD-simulated data: (a) Mean fraction of monomers in the trans state and (b) number of water bridges $n_{\mathrm{wb}}$ in dependence of the force for PEG at different temperatures ranging from $250 \mathrm{~K}$ to $400 \mathrm{~K}$ (with a PEG chain comprising 11 monomers). (c) Schematic picture of a water bridge to PEG in the gauche state with two or one hydrogen involved (left) and a water molecule forming a single hydrogen bond with PEG in the trans state (right). ${ }^{19}$

Figure 6 shows the stretching free energy for PNiPAM vs force for different temperatures determined analogously to the PEG data. In general, AFM experiments and MD simulations agree well. Again, we optimize the coefficient of variation to find the exponent $n$ in the temperature dependence (Figure S11). For the syndiotactic case with MD simulations, a dominance of entropy $(n=1)$ up to $100 \mathrm{pN}$ can be observed (Figure 6b). But then, $n=1 / 3$ describes the course of the temperature dependence of $F / L_{0}$ well, resulting in a dominance of the energetic character. In fact, the syndiotactic case of the MD simulations is quite close to the atactic case of the experimental data. Altogether, we obtain the best fit for $n=1 / 3$, i.e. $F / L_{0} \sim T^{1 / 3}$ (Figure 6c). In general, PNiPAM shows a mixed behaviour $(0<n<1)$ for high stretching forces. 

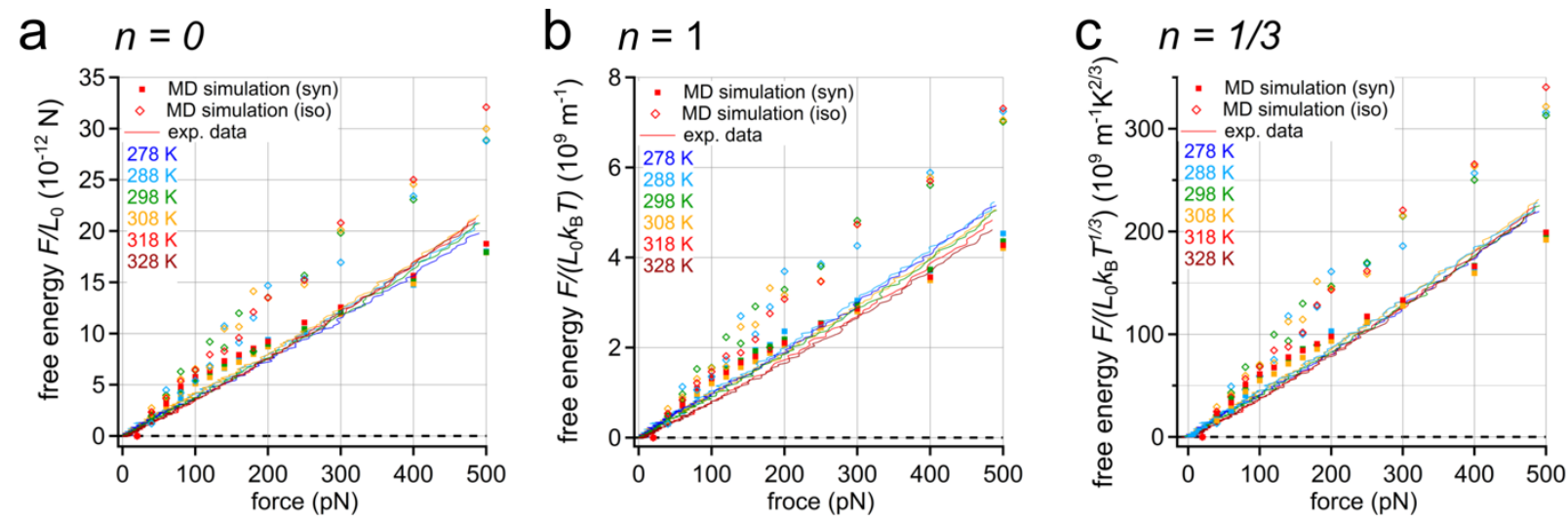

Figure 6. Temperature dependence of the stretching free energy $F$ for the MD-simulations of isotactic PNiPAM and of syndiotactic PNiPAM to experimental AFM data (atactic): (a) stretching free energy $F$ per $L_{0}$ (extension at a force of $500 \mathrm{pN}$ ) vs. force (case $n=0$ ), (b) $F$ per $L_{0}$ divided by $k_{\mathrm{B}} T(n=1)$ and (c) $F$ per $L_{0}$ divided by $k_{\mathrm{B}} T^{1 / 3}(n=1 / 3)$.

At the molecular scale the temperature dependent behavior of PNiPAM can be understood as follows ${ }^{26,60-63}$ : At low temperature, PNiPAM hydrogen bond bridges are formed between water molecules and the amide side groups. Thus, the polymer comprising a hydrophobic backbone is soluble in water. When the temperature is increased, exothermic formation of hydrogen bonds becomes unfavorable. Therefore, the bound water molecules are released leading to a partial dehydration of the polymer. In parallel, intramolecular hydrogen bonding between the amide groups becomes more favorable (attractive interaction). Therefore, the polymer tends to collapse into a globular state, i.e. more force is required to keep the polymer at a certain extension compared to a lower temperature. For single PNiPAM molecules, investigated here, we do not observe a sharp transition, which is rather attributed to cooperative effects as discussed in Futscher et al. ${ }^{27}$, but a gradual change.

The simulation of isotactic PNiPAM (Figure 6a, open rhombi) shows a similar linear relation of free energy $F / L_{0}$ with force. Here, due to the one-sided arrangement of the NiPAM monomer units, the water bridges might be formed between two monomers. This could lead to a greater influence of the water bridges compared to the syndiotactic polymer, where the NiPAM monomer units are arranged in an alternating fashion with respect to the backbone leading to a shallower increase of free energy $F / L_{0}$ with force (Figure 6a, filled squares). Additionally, the magnitude of the 
experimental values for free energy per length $F / L_{0}$ (atactic polymer, Figure 6a, solid lines) is close to the simulated syndiotactic data while the simulated isotactic values are about 1.5 times higher for a given force in the range of $200-500 \mathrm{pN}$. In conclusion, the tactility of PNiPAM is more important for the free energy to stretch the polymer at a given force than a change of temperature from $278 \mathrm{~K}$ to $328 \mathrm{~K}$.

\section{Conclusion}

In a combined experimental and simulation approach, we have scrutinized the temperature dependence of single PEG and PNiPAM polymers. We were able to develop a reliable procedure to covalently attach long polymers (ca. $500 \mathrm{kD}$ ) to the tip of a $\mathrm{Si}_{3} \mathrm{~N}_{4} \mathrm{AFM}$ cantilever and select for single polymers. This allows us to measure highly reproducible single polymer force-extension curves up to high stretching forces (ca. $800 \mathrm{pN}$ ), excluding any interactions with neighboring polymer chains, which is difficult in other (e.g. nanofishing) experiments. Our truly single molecule experiments are consistent with MD simulations in explicit solvent at various temperatures. For PNiPAM we could show that neither the shape nor the frequency of the recorded force-extension curves are significantly changed around the LCST. The LCST is therefore indeed a cooperative effect that only appears at low stretching force.

In addition, we find that PEG and PNiPAM show a contrasting temperature behavior in water. While the stretching force increases with increasing temperature for PNiPAM, a decrease in the stretching force for increasing temperatures can be observed for PEG. The experimental temperature dependent stretching behavior of PEG and PNiPAM is also in good agreement with the MD-simulation.

Furthermore, both experimental data and MD-simulations show a decrease of the force dependent stretching free energy of PEG with increasing temperature for high stretching forces. This can be explained by the influence of temperature on the mean fraction of gauche and trans states of the PEG chain and the corresponding reduction of the number of water bridges during stretching. PNiPAM also shows a non-linear dependence of the free energy per length on temperature.

In summary both, single PEG and PNiPAM molecules, do not constitute purely entropic springs. Their stretching free energy is dominated by energetic solvation effects. This shows that 
although the FJC and WLC models are very helpful to compare polymer properties, the physical interpretation of the resulting parameters has to be done with care. In particular, as has been found before, both models do not mimic the force-extension behaviour over the full range of stretching forces. Here, we could further show that they also do not correctly describe the temperature dependent force-extension behaviour, in particular for highly stretched polymers under aqueous conditions.

We propose to use the exponent $n$ of the temperature dependence of the normalized stretching free energy $\left(F / L_{0}\right)$ as a measure for the degree of energetic vs. entropic character of a polymer. A purely entropic chain is defined by $n=1$, as given in the FJC model. For PNiPAM, we find a mixed behavior with $0<n<1$. For PEG we find a super energetic behavior with $n<0$. Thus, we are able to classify the temperature behavior of different polymers and to show that PEG has a more dominant energetic character than PNiPAM. At the molecular level, both polymers lose hydrogen bonds with the surrounding water. At the same time, for PNiPAM, intermolecular hydrogen bonds are formed that compensate for that loss. Thus, PNiPAM shows an antagonistic temperature dependent force-extension behaviour compared to PEG.

This paves the way to understand temperature responsive polymers and to design block copolymer structures for a tailor-made temperature behavior. Materials comprising blocks with antagonistic temperature dependence could be used for switches or actuators analogous to the bimetallic effect ${ }^{8,64}$ Here pre-stressed material could be used to adjust the force range. In addition, current thermoresponsive materials are often crosslinked polymer hydrogels. ${ }^{65}$ Here small molecular effects might translate to significant macroscopic effects, e.g. in hierarchical structures ${ }^{66}$ or the devices might be implemented at significant pre-stress to increase the temperature response. Finally, some future adaptive force sensors will use polymeric system that can reach or operate in a highly stretched state. ${ }^{67}$

\section{ASSOCIATED CONTENT}

\section{Supporting Information}

The Supporting Information is available free of charge on the ACS Publications website. Supporting Text, Supporting Table S1, Supporting Figures S1-S11, Supporting References. 


\section{AUTHOR INFORMATION}

\section{Corresponding Author}

* Thorsten Hugel, Roland R. Netz and Bizan N. Balzer. Correspondence should be addressed to: bizan.balzer@physchem.uni-freiburg.de

\section{Author Contributions}

M.G., T.H., R.R.N. and B.N.B. designed research. A.K., C.W., K.H., C.R. and R.S. performed the experiments and evaluated the data with support of M.G., T.H., R.R.N. and B.N.B. The manuscript was written by A.K., M.G., T.H., R.R.N. and B.N.B. with support of all authors.

\section{Notes}

The authors declare no competing financial interest.

\section{ACKNOWLEDGMENT}

Financial support by the German Research Foundation via grant SFB 765 (R.R.N.) and grant SFB 863 (T.H.) is gratefully acknowledged. M.G. and C.R. acknowledge partial support in the frame of the LOEWE project iNAPO by the Hessen State Ministry of Higher Education, Research and the Arts.

\section{References}

(1) Bao, Z.; Feng, Y.; Dodabalapur, A.; Raju, V. R.; Lovinger, A. J. High-Performance Plastic Transistors Fabricated by Printing Techniques. Chem. Mater. 1997, 9, 1299-1301.

(2) Bienk, E.J.; Mikkelsen, N.J. Application of advanced surface treatment technologies in the modern plastics moulding industry. Wear 1997, 207, 6-9.

(3) Chen, C.-T.; Suslick, K. S. One-dimensional coordination polymers: Applications to material science. Coord. Chem. Rev. 1993, 128, 293-322.

(4) Gall, K.; Yakacki, C. M.; Liu, Y.; Shandas, R.; Willett, N.; Anseth, K. S. Thermomechanics of the shape memory effect in polymers for biomedical applications. J. Biomed. Mater. Res. A 2005, $73 A, 339-348$.

(5) Keddie, J. L.; Jones, R. A. L.; Cory, R. A. Size-Dependent Depression of the Glass Transition Temperature in Polymer Films. EPL, 27, 59-64. 
(6) Kuroyanagi, S.; Shimada, N.; Fujii, S.; Furuta, T.; Harada, A.; Sakurai, K.; Maruyama, A. Highly Ordered Polypeptide with UCST Phase Separation Behavior. J. Am. Chem. Soc. 2019, 141, $1261-1268$.

(7) Magnusson, J. P.; Khan, A.; Pasparakis, G.; Saeed, A. O.; Wang, W.; Alexander, C. Ionsensitive "isothermal" responsive polymers prepared in water. J. Am. Chem. Soc. 2008, 130, 10852-10853.

(8) Wei, M.; Gao, Y.; Li, X.; Serpe, M. J. Stimuli-responsive polymers and their applications. Polym. Chem. 2017, 8, 127-143.

(9) Huang, H.; Serpe, M. J. Poly(N-isopropylacrylamide) microgel-based etalons for determining the concentration of ethanol in gasoline. J. Appl. Polym. Sci 2015, 132, 24106.

(10) You, Y.-Z.; Kalebaila, K. K.; Brock, S. L.; Oupický, D. Temperature-Controlled Uptake and Release in PNIPAM-Modified Porous Silica Nanoparticles. Chem. Mater. 2008, 20, 3354-3359.

(11) Qian, J.; Wu, F. Thermosensitive PNIPAM semi-hollow spheres for controlled drug release. J. Mater. Chem. B 2013, 1, 3464-3469.

(12) Geisler, M.; Xiao, S.; Puchner, E. M.; Gräter, F.; Hugel, T. Controlling the structure of proteins at surfaces. J. Am. Chem. Soc. 2010, 132, 17277-17281.

(13) Stetter, F. W. S.; Cwiklik, L.; Jungwirth, P.; Hugel, T. Single lipid extraction: the anchoring strength of cholesterol in liquid-ordered and liquid-disordered phases. Biophys. J. 2014, 107, 11671175.

(14) Li, I. T. S.; Walker, G. C. Signature of hydrophobic hydration in a single polymer. Proc Natl Acad Sci U S A 2011, 108, 16527-16532.

(15) Kienle, S.; Liese, S.; Schwierz, N.; Netz, R. R.; Hugel, T. The effect of temperature on singlepolypeptide adsorption. Chemphyschem 2012, 13, 982-989.

(16) Cui, S.; Pang, X.; Zhang, S.; Yu, Y.; Ma, H.; Zhang, X. Unexpected Temperature-Dependent Single Chain Mechanics of Poly(N-isopropyl-acrylamide) in Water. Langmuir 2012, 28, 51515157. 
(17) Liang, X.; Nakajima, K. Nanofishing of a Single Polymer Chain: Temperature-Induced CoilGlobule Transition of Poly(N -isopropylacrylamide) Chain in Water. Macromol. Chem. Phys. 2018, 219, 1700394.

(18) Zhang, W.; Zou, S.; Wang, C.; Zhang, X. Single Polymer Chain Elongation of Poly(N isopropylacrylamide) and Poly(acrylamide) by Atomic Force Microscopy. J. Phys. Chem. B 2000, $104,10258-10264$.

(19) Liese, S.; Gensler, M.; Krysiak, S.; Schwarzl, R.; Achazi, A.; Paulus, B.; Hugel, T.; Rabe, J. P.; Netz, R. R. Hydration Effects Turn a Highly Stretched Polymer from an Entropic into an Energetic Spring. ACS Nano 2017, 11, 702-712.

(20) Heskins, M.; Guillet, J. E. Solution Properties of Poly(N-isopropylacrylamide). J. Macromol. Sci. A 1968, 2, 1441-1455.

(21) Mao, H.; Li, C.; Zhang, Y.; Bergbreiter, D. E.; Cremer, P. S. Measuring LCSTs by novel temperature gradient methods: evidence for intermolecular interactions in mixed polymer solutions. J. Am. Chem. Soc. 2003, 125, 2850-2851.

(22) Deshmukh, S. A.; Sankaranarayanan, S. K. R. S.; Suthar, K.; Mancini, D. C. Role of Solvation Dynamics and Local Ordering of Water in Inducing Conformational Transitions in Poly(Nisopropylacrylamide) Oligomers through the LCST. J. Phys. Chem. B 2012, 116, 2651-2663.

(23) Chung, J.E.; Yokoyama, M.; Yamato, M.; Aoyagi, T.; Sakurai, Y.; Okano, T. Thermoresponsive drug delivery from polymeric micelles constructed using block copolymers of poly(Nisopropylacrylamide) and poly(butylmethacrylate). J. Control. Release 1999, 62, 115-127.

(24) Okano, T.; Bae, Y. H.; Jacobs, H.; Kim, S. W. Thermally on-off switching polymers for drug permeation and release. J. Control. Release 1990, 11, 255-265.

(25) Kato, D.; Sohn, W. Y.; Katayama, K. Aggregation-Induced Expansion of Poly-(N -isopropyl acrylamide) Solutions Observed Directly by the Transient Grating Imaging Technique. ACS Omega 2018, 3, 8484-8490.

(26) Abbott, L. J.; Tucker, A. K.; Stevens, M. J. Single Chain Structure of a Poly(Nisopropylacrylamide) Surfactant in Water. J. Phys. Chem. B 2015, 119, 3837-3845. 
(27) Futscher, M. H.; Philipp, M.; Müller-Buschbaum, P.; Schulte, A. The Role of Backbone Hydration of Poly(N-isopropyl acrylamide) Across the Volume Phase Transition Compared to its Monomer. Sci. Rep. 2017, 17012, 1-10.

(28) Kutnyanszky, E.; Embrechts, A.; Hempenius, M. A.; Vancso, G. J. Is there a molecular signature of the LCST of single PNIPAM chains as measured by AFM force spectroscopy? Chemical Physics Letters 2012, 535, 126-130.

(29) Harnoy, A. J.; Rosenbaum, I.; Tirosh, E.; Ebenstein, Y.; Shaharabani, R.; Beck, R.; Amir, R. J. Enzyme-Responsive Amphiphilic PEG-Dendron Hybrids and Their Assembly into Smart Micellar Nanocarriers. J. Am. Chem. Soc. 2014, 136, 7531-7534.

(30) Duncan, R. Polymer conjugates as anticancer nanomedicines. Nat. Rev. Cancer 2006, 6, 688 $-701$.

(31) Chen, J.; Spear, S. K.; Huddleston, J. G.; Rogers, R. D. Polyethylene glycol and solutions of polyethylene glycol as green reaction media. Green Chem. 2005, 7, 64-82.

(32) Brandenberger, C.; Mühlfeld, C.; Ali, Z.; Lenz, A.-G.; Schmid, O.; Parak, W. J.; Gehr, P.; Rothen-Rutishauser, B. Quantitative Evaluation of Cellular Uptake and Trafficking of Plain and Polyethylene Glycol-Coated Gold Nanoparticles. Small 2010, 6, 1669-1678.

(33) Kjellander, R.; Florin, E. Water structure and changes in thermal stability of the system poly(ethylene oxide)-water. J. Chem. Soc., Faraday Trans. 1 1981, 77, 2053-2077.

(34) Balzer, B. N.; Hugel, T. 2.25 - Single-Molecule Detection and Manipulation. In Polymer science: A comprehensive reference; Matyjaszewski, K., Möller, M., Eds.; Elseiver: Amsterdam, 2012; pp 629-645.

(35) Oesterhelt, F.; Rief, M.; Gaub, H. E. Single molecule force spectroscopy by AFM indicates helical structure of poly(ethylene-glycol) in water. New. J. Phys. 1999, 1, 6.1 - 6.11.

(36) Bartell, L. S. On the Effects of Intramolecular van der Waals Forces. J. Chem. Phys. 1960, $32,827-831$.

(37) Hess, B.; Kutzner, C.; van der Spoel, D.; Lindahl, E. GROMACS 4: Algorithms for Highly Efficient, Load-Balanced, and Scalable Molecular Simulation. J. Chem. Theory Comput. 2008, 4, $435-447$. 
(38) Bussi, G.; Donadio, D.; Parrinello, M. Canonical sampling through velocity rescaling. J. Chem. Phys. 2007, 126, 14101.

(39) Parrinello, M.; Rahman, A. Polymorphic transitions in single crystals: A new molecular dynamics method. J. Appl. Phys. 1998, 52, 7182-7190.

(40) Essmann, U.; Perera, L.; Berkowitz, M. L.; Darden, T.; Lee, H.; Pedersen, L. G. A smooth particle mesh Ewald method. J. Chem. Phys. 1998, 103, 8577-8593.

(41) Lee, H.; Venable, R. M.; Mackerell, A. D.; Pastor, R. W. Molecular dynamics studies of polyethylene oxide and polyethylene glycol: hydrodynamic radius and shape anisotropy. Biophys. J. 2008, 95, 1590-1599.

(42) Berendsen, H. J. C.; Grigera, J. R.; Straatsma, T. P. The missing term in effective pair potentials. J. Phys. Chem. 1987, 91, 6269-6271.

(43) Jorgensen, W. L.; Tirado-Rives, J. The OPLS optimized potentials for liquid simulations potential functions for proteins, energy minimizations for crystals of cyclic peptides and crambin. J. Am. Chem. Soc. 1988, 110, 1657-1666.

(44) Kanduč, M.; Chudoba, R.; Palczynski, K.; Kim, W. K.; Roa, R.; Dzubiella, J. Selective solute adsorption and partitioning around single PNIPAM chains. Phys. Chem. Chem. Phys. 2017, 19, 5906-5916.

(45) Palivec, V.; Zadrazil, D.; Heyda, J. All-atom REMD simulation of poly-Nisopropylacrylamide thermodynamics in water: a model with a distinct 2-state behavior. Cornell University Library 2018, arXiv:1806.05592, arXiv preprint.

(46) Pirzer, T.; Hugel, T. Atomic force microscopy spring constant determination in viscous liquids. Rev. Sci. Instrum. 2009, 80, 35110.

(47) Walder, R.; LeBlanc, M.-A.; van Patten, W. J.; Edwards, D. T.; Greenberg, J. A.; Adhikari, A.; Okoniewski, S. R.; Sullan, R. M. A.; Rabuka, D.; Sousa, M. C.; Perkins, T. T. Rapid Characterization of a Mechanically Labile $\alpha$-Helical Protein Enabled by Efficient Site-Specific Bioconjugation. J. Am. Chem. Soc. 2017, 139, 9867-9875.

(48) Barradas, R. G.; Fletcher, S.; Porter, J. D. The hydrolysis of maleimide in alkaline solution. Can. J. Chem. 1976, 54, 1400-1404. 
(49) Hutter, J. L.; Bechhoefer, J. Calibration of atomic-force microscope tips. Rev. Sci. Instrum. 1998, 64, 1868-1873.

(50) Trathnigg, B. Determination of MWD and chemical composition of polymers by chromatographic techniques. Prog. Polym. Sci. 1995, 20, 615-650.

(51) Blass, J.; Albrecht, M.; Wenz, G.; Zang, Y. N.; Bennewitz, R. Single-molecule force spectroscopy of fast reversible bonds. Phys. Chem. Chem. Phys. 2017, 19, 5239-5245.

(52) Serr, A.; Netz, R. R. Pulling adsorbed polymers from surfaces with the AFM: stick vs. slip, peeling vs. gliding. EPL 2005, 73, 292.

(53) Balzer, B. N.; Gallei, M.; Hauf, M. V.; Stallhofer, M.; Wiegleb, L.; Holleitner, A.; Rehahn, M.; Hugel, T. Nanoscale friction mechanisms at solid-liquid interfaces. Angew. Chem. Int. Edit. 2013, 52, 6541-6544.

(54) Fava, A.; Iliceto, A.; Camera E. Kinetics of the Thiol-Disulfide Exchange 1956, J. Am. Chem. Soc., 833-838.

(55) Marchand, P.; Marmet, L. Binomial smoothing filter: A way to avoid some pitfalls of leastsquares polynomial smoothing. Rev. Sci. Instrum. 1983, 54, 1034-1041.

(56) Li, B.; Wang, X.; Li, Y.; Paananen, A.; Szilvay, G. R.; Qin, M.; Wang, W.; Cao, Y. SingleMolecule Force Spectroscopy Reveals Self-Assembly Enhanced Surface Binding of Hydrophobins. Chemistry - A European Journal 2018, 24, 9224-9228.

(57) Idota, N.; Nagase, K.; Tanaka, K.; Okano, T.; Annaka, M. Stereoregulation of Thermoresponsive Polymer Brushes by Surface-Initiated Living Radical Polymerization and the Effect of Tacticity on Surface Wettability. Langmuir 2010, 26, 17781-17784.

(58) Smith, S. B.; Finzi, L.; Bustamante, C. Direct Mechanical Measurements of the Elasticity of Single DNA Molecules by Using Magnetic Beads. Science 1992, 258, 1122-1126.

(59) Marko, J. F.; Siggia, E. D. Stretching DNA. Macromolecules 1995, 28, 8759-8770.

(60) Katsumoto, Y.; Tanaka, T.; Sato, H.; Ozaki, Y. Conformational change of poly(Nisopropylacrylamide) during the coil-globule transition investigated by attenuated total reflection/infrared spectroscopy and density functional theory calculation. The Journal of Physical Chemistry A 2002, 106, 3429-3435. 
(61) Lin, S. Y.; Chen, K. S.; Liang, R. C. Thermal micro ATR/FT-IR spectroscopic system for quantitative study of the molecular structure of poly(N-isopropylacrylamide) in water. Polymer 1999, 40, 2619-2624.

(62) Maeda, Y.; Nakamura, T.; Ikeda, I. Changes in the Hydration States of Poly(Nalkylacrylamide)s during Their Phase Transitions in Water Observed by FTIR Spectroscopy \dag. Macromolecules 2001, 34, 1391-1399.

(63) Percot, A.; Zhu, X. X.; Lafleur, M. A simple FTIR spectroscopic method for the determination of the lower critical solution temperature of $\mathrm{N}$-isopropylacrylamide copolymers and related hydrogels. J. Polym. Sci. B Polym. Phys. 2000, 38, 907-915.

(64) Chen, J.; Liu, M.; Gong, H.; Huang, Y.; Chen, C. Synthesis and Self-Assembly of Thermoresponsive PEG- b-PNIPAM- b-PCL ABC Triblock Copolymer through the Combination of Atom Transfer Radical Polymerization, Ring-Opening Polymerization, and Click Chemistry. $J$. Phys. Chem. B 2011, 115, 14947-14955.

(65) Es Sayed, J.; Lorthioir, C.; Perrin, P.; Sanson, N. PEGylated NiPAM microgels: synthesis, characterization and colloidal stability. Soft Matter 2019, 15, 963-972.

(66) Zhu, B.; Jasinski, N.; Benitez, A.; Noack, M.; Park, D.; Goldmann, A. S.; Barner-Kowollik, C.; Walther, A. Hierarchical Nacre Mimetics with Synergistic Mechanical Properties by Control of Molecular Interactions in Self-Healing Polymers. Angewandte Chemie 2015, 127, 8777-8781.

(67) Merindol, R.; Delechiave, G.; Heinen, L.; Catalani, L. H.; Walther, A. Modular Design of Programmable Mechanofluorescent DNA Hydrogels. Nature Communications 2019, 10, 1-10. 